\title{
Compression of RADARSAT Data with Block Adaptive Wavelets
}

\author{
Ian Cumming and Jing Wang \\ Department of Electrical and Computer Engineering \\ The University of British Columbia \\ 2356 Main Mall, Vancouver, BC, Canada V6T1Z4 \\ ianc@ece.ubc.ca jingwa@ece.ubc.ca \\ Tel: (604) 822-4623 Fax: (604) 822-5949
}

\begin{abstract}
This paper proposes a new algorithm referred to as the Wavelet Packet-based Embedded Block coding (WPEB) scheme for SAR data compression. This algorithm combines the following properties: (1) wavelet packet decomposition is adopted to exploit middle and high frequency components associated with SAR data; (2) block coding is utilized to improve DWT coefficient coding efficiency by adaptively allocating more bits to regions of importance with higher information content (e.g. more contrast, edges); (3) speckle reduction is built into the bit allocation scheme by using wavelet transform denoising. Examples are given using RADARSAT data, which show that the compression performance is better than conventional wavelet methods and visual image interpretation is acceptable at $1 \mathrm{bpp}$.
\end{abstract}

\section{Introduction}

SAR image data can provide unique information about the surface of the Earth [1]. The volume of data associated with Earth information is so huge that the compression of SAR data becomes crucial to transmission and archiving.

At present, most image compression algorithms are designed for standard test images, usually optical images $[2,4,5]$. The most popular algorithms for still image compression are those included in the JPEG/JPEG2000 standard. These algorithms are designed to compress traditional images, and do not lead to ideal compression results for SAR images. This is because these compression schemes are not designed to account for SAR data characteristics, such as high dynamic range, the speckle phenomena, and the presence of significant high frequency components arising from terrain texture and edges.

WPEB belongs to the class of lossy transform compression algorithms. Our coding scheme includes the wavelet packet transform [3], embedded block coding, speckle reduction, and optimal bit allocation. We consider the SAR data spectrum in wavelet analysis and bit allocation in order to achieve better compression performance and make this algorithm more flexible when compressing different types of SAR data (ocean, snow, ice, city, forest, agriculture, mountains, etc), and satisfying different compression evaluation criteria.

A block diagram for WPEB encoding and decoding is shown in Figure 1. At the encoder, the discrete wavelet packet transform is applied to the source image after it is divided into blocks. The transform coefficients are then quantized and entropy coded to form the output sequence. The decoder is the reverse of the encoder, consisting of entropy decoding, inverse quantization, and an inverse wavelet packet transform. 


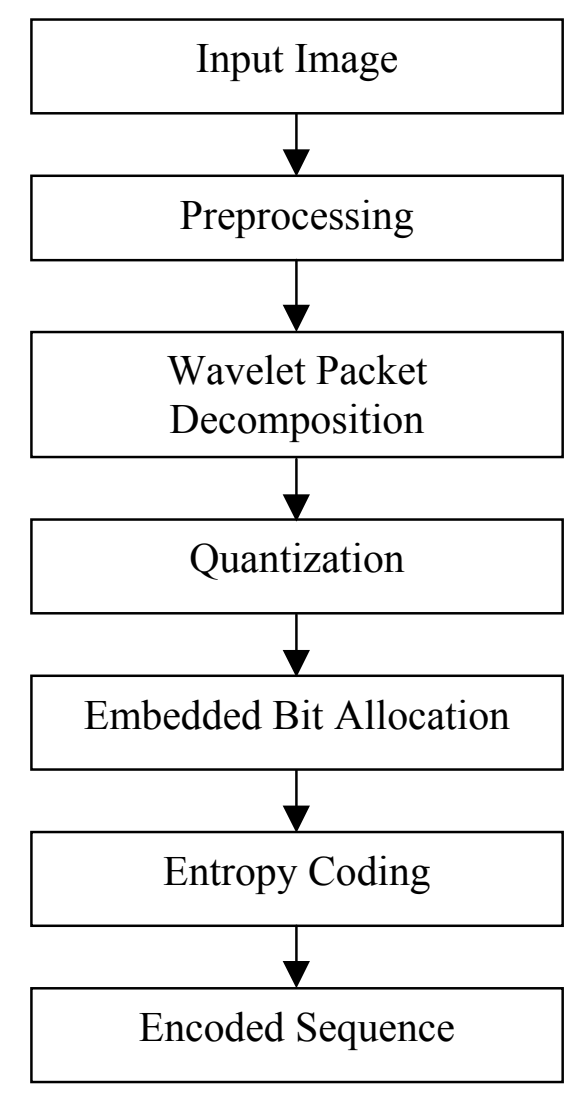

(a) Encoding

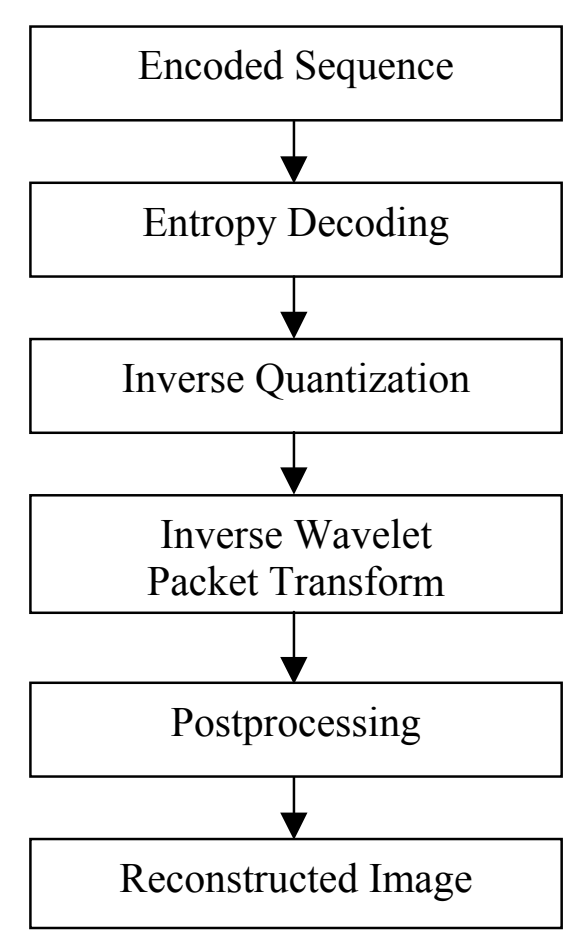

(b) Decoding

Figure 1. Block diagram of WPEB compression algorithm.

\section{Wavelet Packet Decomposition}

SAR data usually have significant middle and high frequency components, as in regions with textures and edges; this makes the wavelet packet transform a better choice than the standard discrete wavelet transform (DWT) for SAR data compression. Wavelet packet decomposition differs from the standard wavelet transform by allowing the decomposition of the upper frequency bands as well as the lower ones. By this method, wavelet packets can be used to achieve a more accurate representation and compression of the medium and high frequency information in SAR images.

In previous work of SAR image compression [6], texture analysis is based on subband energy at different decomposition levels. The total energy $E_{S B}$ of each sub-band is defined as:

$$
E_{s b}=\frac{1}{M N} \sum_{m=1}^{M} \sum_{n=1}^{N}|x(m, n)|^{2}
$$

where $\mathrm{x}(\mathrm{m}, \mathrm{n})$ is the wavelet coefficient set at a given decomposition level. Because the low-low sub-band normally has the maximum energy, we call its energy $E_{\text {ref }}$ and compare the energy of other sub-bands with it. A constant $\mathrm{C}_{\mathrm{sb}}<1$ is used as the criterion for 
applying another level of wavelet packet decomposition. When the energy $\mathrm{E}_{\mathrm{sb}}$ of a given sub-band is greater than $\mathrm{C}_{\mathrm{SB}} \mathrm{E}_{\text {ref, }}$, wavelet packets are applied to further decompose the higher frequencies.

For our algorithm, a biorthogonal wavelet [3] is selected because of its linear phase property, and because edge symmetry can be obtained using symmetrical extension. Since all test images are normalized to a zero mean before the DWT is applied, the energy measure $\mathrm{E}_{\mathrm{SB}}$ is simply the sub-band variance. At each decomposition stage, the wavelet packet analysis proceeds as follows:

(1) Initialize $C_{H L}, C_{L H}, C_{H H}$, and set the maximum decomposition level.

(2) Apply a DWT, to obtain the four sub-band coefficients, LL, LH, HL and HH.

(3) Calculate the variance for each sub-band, $\mathrm{E}_{\mathrm{SB}}$.

(4) If $\mathrm{E}_{\mathrm{LH}}>\mathrm{C}_{\mathrm{LH}} \mathrm{E}_{\text {ref, }}$, then apply a DWT to LH.

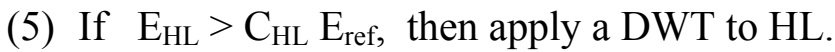

(6) If $\mathrm{E}_{\mathrm{HH}}>\mathrm{C}_{\mathrm{HH}} \mathrm{E}_{\mathrm{ref}}$, then apply a DWT to $\mathrm{HH}$.

(7) Apply a DWT to LL.

(8) If the decomposition level is reached, stop; otherwise go to step (3).

\section{Embedded Block Coding}

It is known that block transform coders enjoy success because of their low complexity and their effective performance. The most popular block transform coder is JPEG, which utilizes the $8 \times 8$ Discrete Cosine Transform (DCT).

The general idea behind block coding is that the image can be divided into blocks, so that wavelet decomposition, quantization and coding can be applied to smaller sections of the image, rather than to the whole image at once. In this way, different number of bits can be allocated to each block so that the overall image SNR is maximized.

Generally, large image blocks lead to smaller mean squared error in the sense that the correlation within larger block samples can be exploited more fully in compression. This is true for optical images with correlation coefficients as high as 0.95 , but not so true for SAR images where the correlation coefficients are usually below 0.7 .

The advantages of using block coding include the following:

(1) Different statistics of each block can be recognized. The statistics, such as dynamic data range and entropy, affect coding performance in the sense that the data range is associated with quantization steps, and entropy is associated with the maximum compression ratio for a given image block.

(2) The flexibility of assigning different number of bits to each block is possible, within the constraint of a specified total number of bits per image.

On the other hand, the disadvantages of blocking include discontinuities and artifacts across the block boundaries, especially at very low bit rates. The choice of block size is a compromise between (1) obtaining good compression within a block and avoiding block artifacts (favors larger block sizes), and (2) adaptation to block statistics 
and computing efficiency (favors smaller block sizes). In practice, we found that a block size between 128 and 256 to be appropriate for SAR images.

Generally, we apply an embedded blocking algorithm by designing a bit allocation scheme to adaptively assign different numbers of bits to image regions based on importance. This is a practical problem when dealing with images containing many types of scene features. For example, if the SAR image is used for sea-ice classification, an ideal compression scheme preserves texture regions that represent different types of ice (first year ice, multi year ice, etc.). Some regions of an image contain features not of interest, such as land areas in sea ice images, and thus we can assign fewer bits to these regions.

\section{Bit Allocation Scheme With Speckle Reduction}

In this subsection, we explain the bit allocation problem mathematically. Since the image is composed of a collection of coded blocks $B_{i}$, with embedded bit streams that may be truncated at a rate $R_{i}^{n}$, the corresponding contribution to the distortion in the reconstructed image is denoted as $D_{i}^{n}$ for each truncation point $n_{i}$. The relevant distortion matrix is additive:

$$
D=\sum_{i} D_{i}^{n_{i}}
$$

where $\mathrm{D}$ is the total distortion for the reconstructed image, and $n_{i}$ is the truncation level giving $B_{i}$ bits for block $i$. From the rate distortion optimization point of view, the problem is to find the optimal selection of $n_{i}$ so as to minimize the overall distortion $\mathrm{D}$, subject to a constraint, $\mathrm{R}$, where $\mathrm{R}$ is the allowed bit rate for the whole image.

If the image is compressed as a single block, the overall distortion is equal to the block distortion. For a specific encoding algorithm, the rate-distortion curve is monotonic with only one parameter, the compression ratio or the truncation point $n_{i}$, as shown in Figure 2(a).

If the image is divided into two blocks, then overall optimization can be obtained by searching the minimum MSE as shown in Figure 2(b). If $b_{p} p_{\mathrm{T}}$ is the total allowed bpp, and $\mathrm{bpp}_{i}$ is the bpp of block $i$, then we search for the optimal $\mathrm{bpp}_{1}$ given the constraint that

$$
\mathrm{bpp}_{\mathrm{T}}=\mathrm{bbp}_{1}+\mathrm{bpp}_{2}
$$

The vertical axis in Figure 2(b) is the distortion for the whole image at a given $\mathrm{bpp}_{\mathrm{T}}$. It is interesting to note that the distortion curve for a specific compression ratio for the whole image is a "U" shape, with a unique minimum. The U shape arises because for each block, the rate-distortion curve is convex, which means the slope of the curve decreases as the bpp value increases. Therefore for the overall distortion curve, the slope decreases on the left side of the $U$ shape, and increases on the right side of the $U$ shape. In other words, if we assign too many bits (or too few bits) to block 1, the overall compression performance will drop. Also there is no guarantee the $U$ shape is symmetric. Symmetry occurs when two blocks have the same rate-distortion curve. 


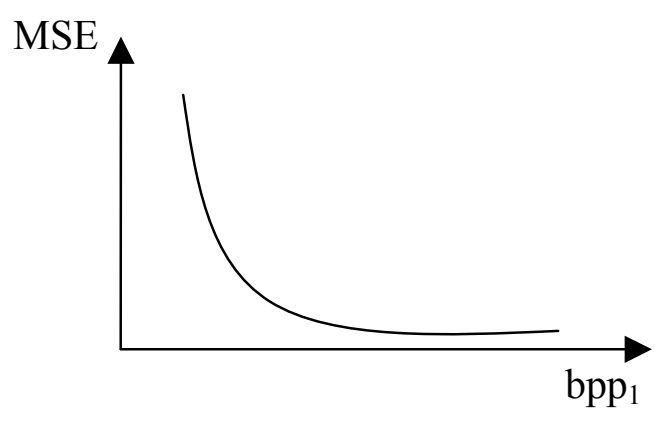

(a) one block is used

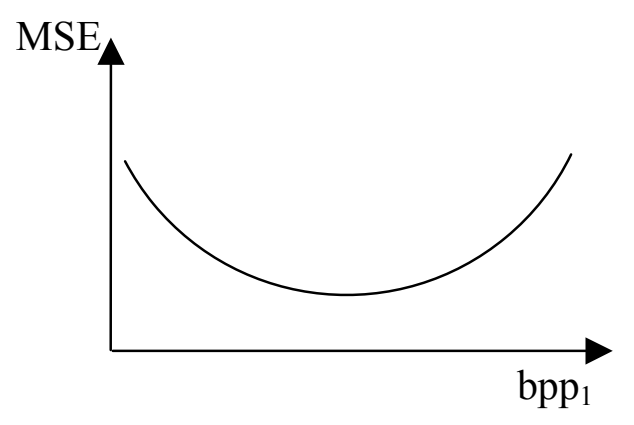

(b) two blocks are used

Figure 2. Rate-distortion curves for one and two blocks.

Another parameter affecting bit allocation is speckle reduction. We use hardthresholding to reduce speckle, depending upon the block dynamic range [7]. For blocks with high speckle noise, more aggressive hard thresholding reduces the number of bits used.

\subsection{Blocks of Importance}

Blocks that contain important features for classification or detection should be assigned more bits. If $\left\{\mathrm{B}_{1}, \mathrm{~B}_{2}, \ldots \mathrm{B}_{\mathrm{n}}\right\}$ is the number of bits for each image block, and $\mathrm{B}_{\mathrm{T}}$ is the total number of bits specified by $\mathrm{R}$, then:

$$
\mathrm{B}_{\mathrm{T}}=\mathrm{B}_{1}+\mathrm{B}_{2}+\ldots+\mathrm{Bn}_{\mathrm{n}}
$$

Suppose that each block is the same size, and that each is compressed at the same ratio; then we could expect $\mathrm{B}_{1}=\mathrm{B}_{2}=\ldots=\mathrm{B}_{\mathrm{n}}$. However, it is possible in some applications (such as the "region of interest" feature in JPEG2000) that the number of bits assigned to each block differs, especially when different image blocks have completely different local statistics.

\subsection{Dynamic Data Range}

As each block can have a different dynamic range, we define a Data Dynamic Range (DDR) set $\left\{\mathrm{d}_{1}, \mathrm{~d}_{2}, \ldots \mathrm{d}_{\mathrm{n}}\right\}$ to represent this information. Since the wavelet transform is a linear, the image block that has a large data range also contains a large range of wavelet coefficients. In order to encode these coefficients with as little distortion as possible, we need to increase the quantization level by decreasing the decision step, and allocate more bits to this block.

\section{Experimental Results}

Experiments are done on a test image shown in Figure 5(a). The raw data were acquired in February 1998 by RADARSAT-1, and processed in July 1999 by Radarsat International. The scene includes Vancouver airport and surrounding urban areas. The comparison between the WPEB and SPIHT algorithms [4] is used to demonstrate the merits of our compression algorithm. 
In the experiment, the test image of $512 \times 1024$ pixels is divided into two blocks of the same size $(512 \times 512)$. The variance of the left block is 1330 , while the variance of the right block is 5340, and the variance for the whole image is 4350 .

\subsection{Bit Allocation Results}

The optimal bit allocation can be obtained by searching the minima of the ratedistortion curve at specific overall compression ratios, as shown in Figure 3. The search results are shown in Table 1. It was found that the optimal compression ratio for each block is not the same as for the whole image for the 2-block scene.

In practice, the search is inefficient when the image is divided into many blocks. However, it was found that a simple statistical measure such as block standard deviation was able to give a near-optimal bit allocation.

Table 1 Optimal compression ratio for two blocks

\begin{tabular}{|l|l|l|l|l|}
\hline Overall & $0.5 \mathrm{bpp}$ & $1.0 \mathrm{bpp}$ & $1.5 \mathrm{bpp}$ & $2.0 \mathrm{bpp}$ \\
\hline Left block & $0.3 \mathrm{bpp}$ & $0.6 \mathrm{bpp}$ & $1.1 \mathrm{bpp}$ & $1.6 \mathrm{bpp}$ \\
\hline Right block & $0.7 \mathrm{bpp}$ & $1.4 \mathrm{bpp}$ & $1.9 \mathrm{bpp}$ & $2.4 \mathrm{bpp}$ \\
\hline
\end{tabular}
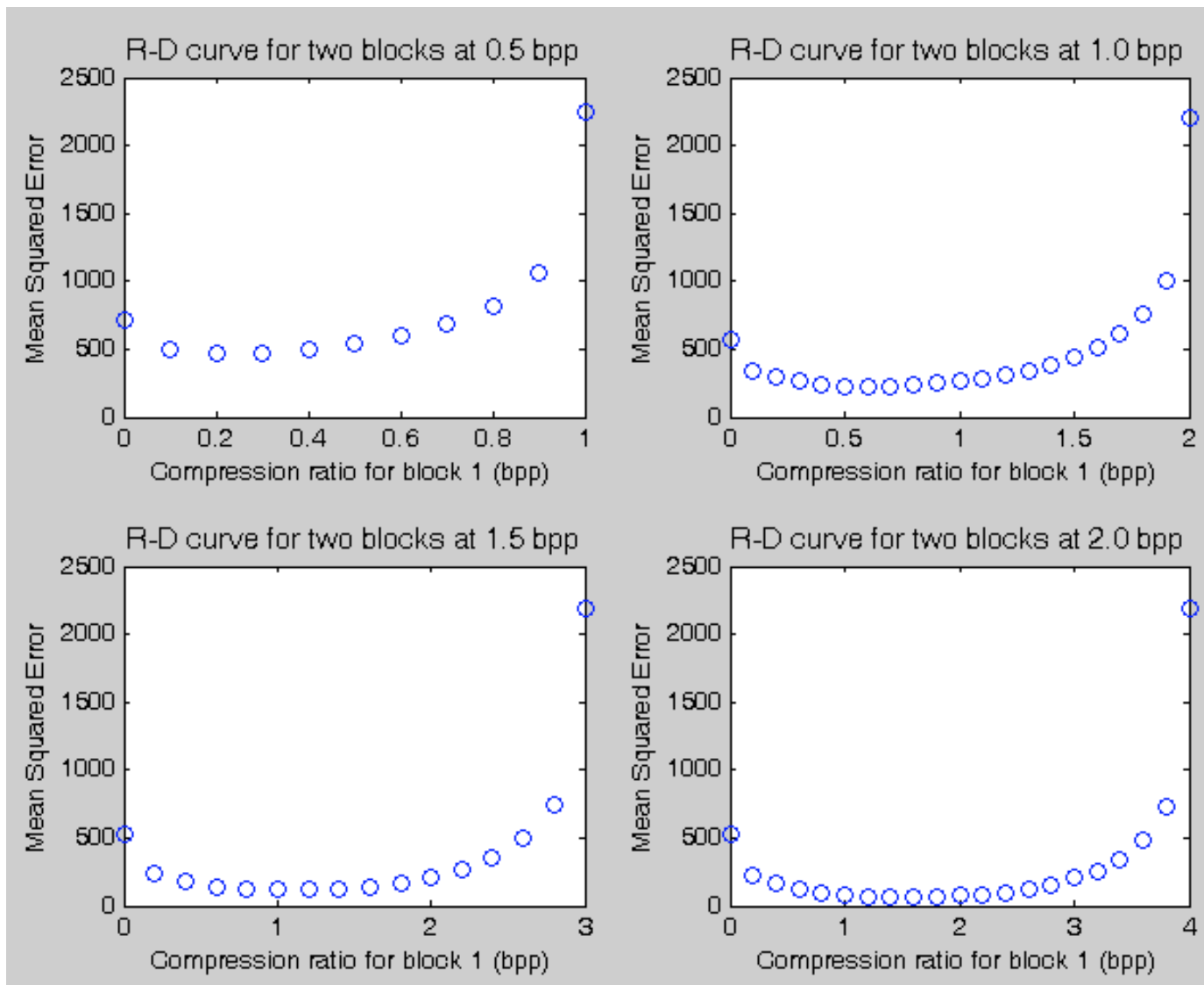

Figure 3. Search for the optimal bit allocation between two blocks. 
The four curves in Figure 3 show the rate-distortion curves for average bit rates of $0.5 \mathrm{bpp}, 1.0 \mathrm{bpp}, 1.5 \mathrm{bpp}, 2.0 \mathrm{bpp}$, respectively. The horizontal axis shows the compression ratio for Block 1, and the vertical axis is the MSE for the whole image. The bpp value for Block 2 is given by equation (3).

\subsection{MSE Results}

In order to compare the performance of the WPEB algorithm fairly, it is useful to compare it against the SPIHT algorithm applied over the whole image, and the SPIHT applied using two blocks (but with the same number of bits in each block). The MSE results for the SPIHT, averaged 2-block SPIHT (ASPIHT) and WPEB algorithms are shown in Figure 4. The MSE for WPEB is 25\% lower than that for 1-block SPIHT, and $12 \%$ lower than the 2-block SPIHT. The improvement of the 2-block SPIHT over the 1block SPIHT is further evidence of the advantages of blocking.

The reconstructed images at $1.0 \mathrm{bpp}$ for the SPIHT and WPEB algorithms are shown in Figure 5. The WPEB image is visually better than the SPIHT image. In the left block, more details are observed in the water in the WPEB case, even though fewer bits are used. Also block artifacts can be seen in the water in the SPIHT case. In the right block, the city and airport details of the WPEB case are closer to those in the original image than the SPIHT case.

\section{Conclusions}

Our compression algorithm has two main strengths. First, we use wavelet packet decomposition to better represent SAR imagery's significant middle and high frequency components. Second, we utilize a block coding scheme to exploit statistical properties, such as the activity level or energy compaction, of each block. Our experimental results show that this coding scheme gives a lower MSE than traditional wavelet methods and is promising for SAR image compression.

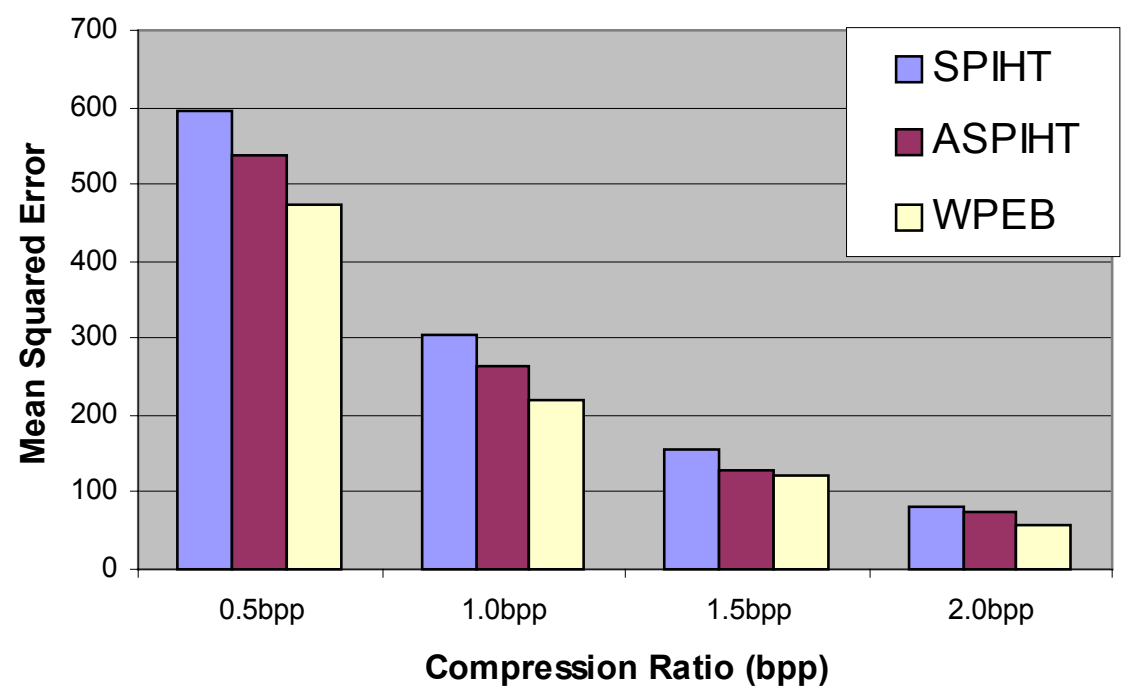

Figure 4. MSE results of SPIHT and WPEB encoding of the test image. 


\section{References}

[1] C. Oliver, S. Quegan. Understanding Synthetic Aperture Radar Images. Artech House, 1998.

[2] K. Sayood. Introduction to Data Compression, $2^{\text {nd }}$ edition. Academic Press, USA, 2000.

[3] C. S. Burrus, R. A. Gopinath, H. Guo. Introduction to Wavelets and Wavelet Transforms. Prentice-Hall, 1998.

[4] A. Said, W. A. Pearlman. A New Fast and Efficient Image Codec Based on Set Partitioning in Hierarchical Trees. IEEE Transactions on Circuits and Systems for Video Technology, vol. 6, no. 3, pp. 243-250, June 1996.

[5] D. Taubman. High Performance Scalable Image Compression with EBCOT. IEEE Transactions on Image Processing, vol. 9, no. 7. pp. 1158-1170, July, 2000.

[6] Z. Zeng, I Cumming. SAR Image Compression Using a Tree-Structured Wavelet Transform. IEEE Transactions on Geoscience and Remote Sensing, vol. 39, no. 3, pp. 546-552, 2001.

[7] D. Donoho. De-Noising by Soft-Thresholding. IEEE Transactions on Information Theory, vol. 41, no. 3, pp. 613-627, May 1995. 


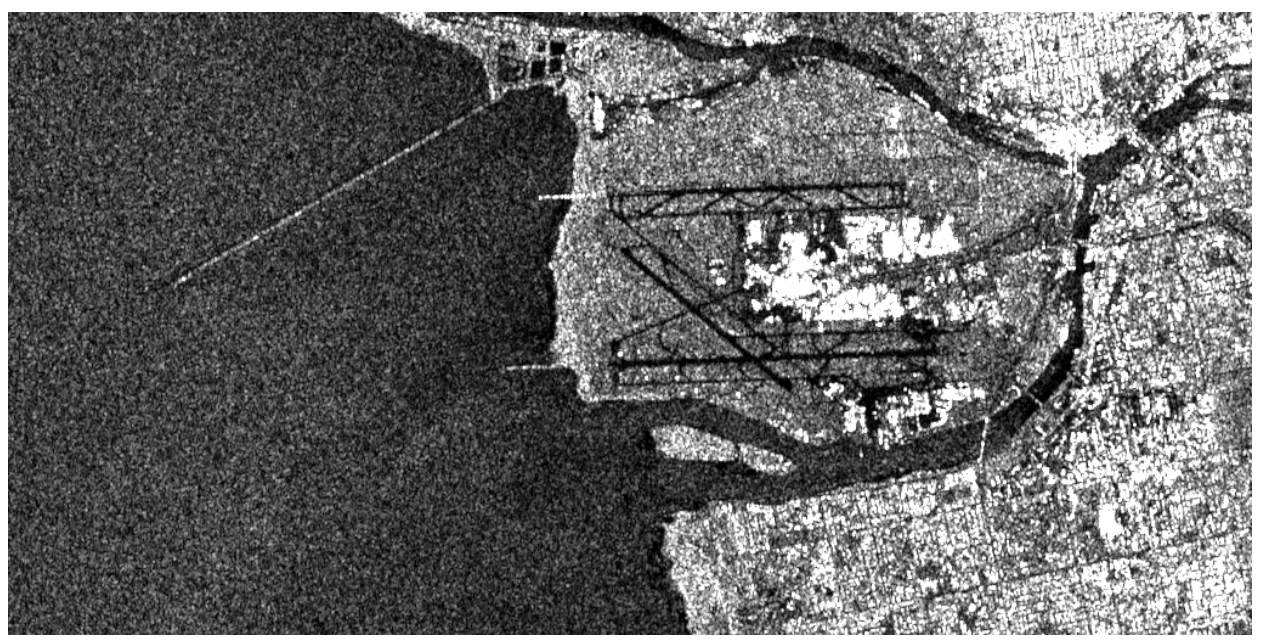

(a) Original RADARSAT-1 image of Vancouver airport

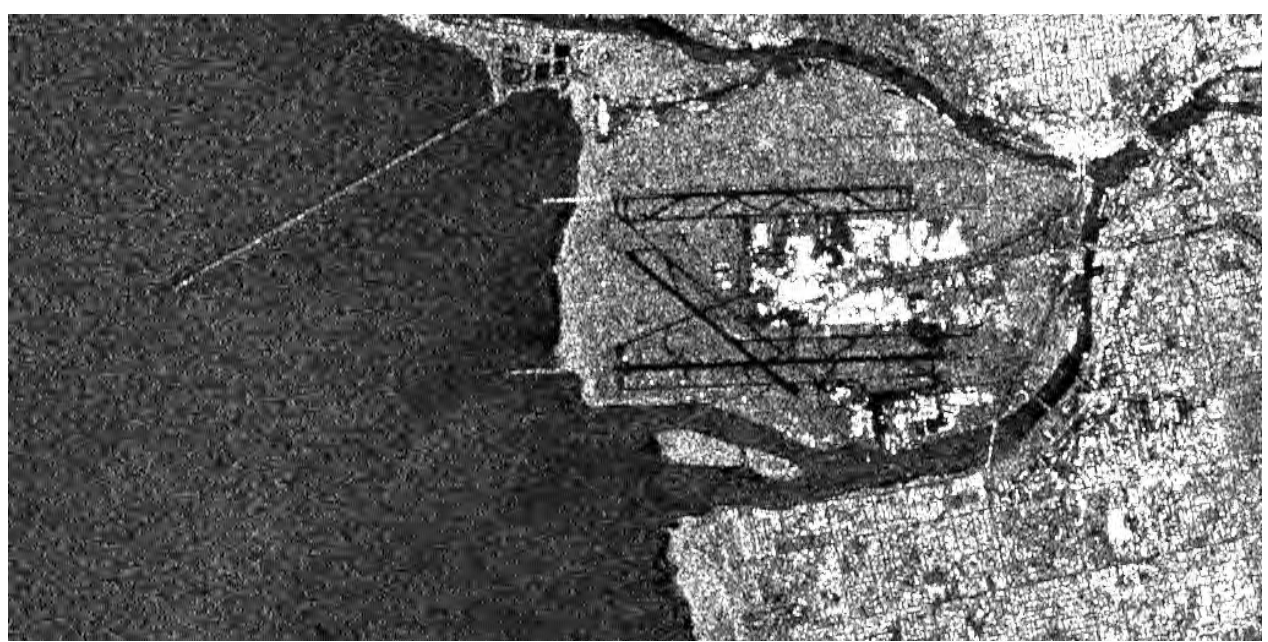

(b) SPIHT algorithm

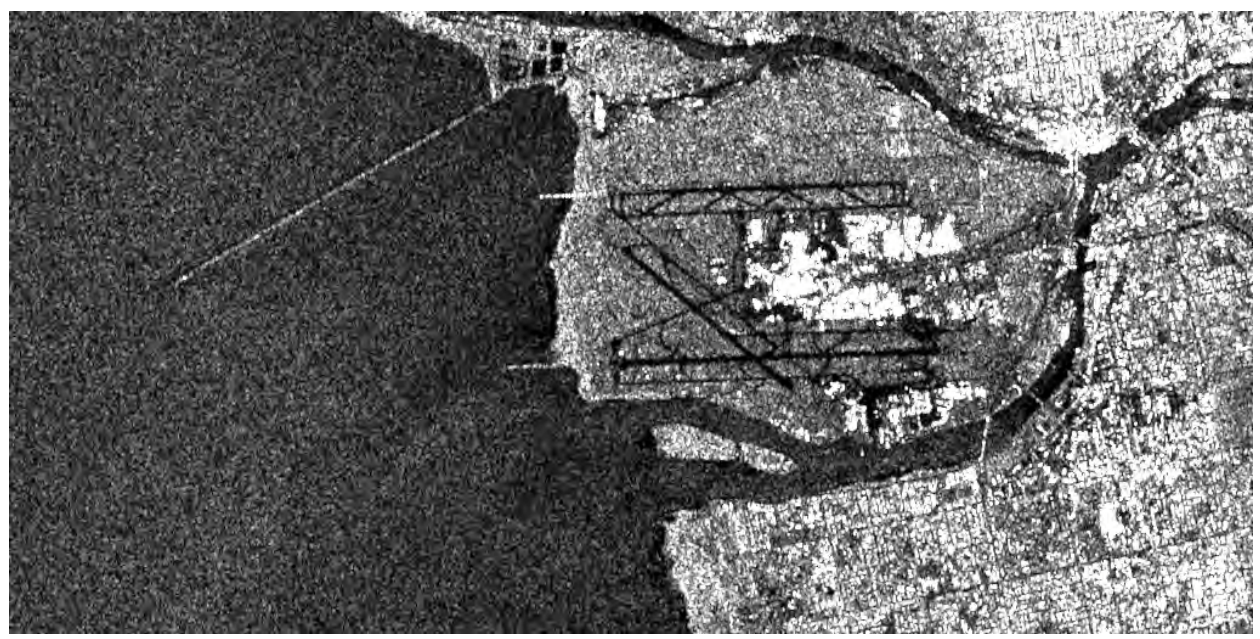

(c) WPEB algorithm

Figure 5. Compression results for SPIHT and WPEB at $1.0 \mathrm{bpp}$. 